\title{
Integration of cardiac responses to serial stimuli after Pavlovian conditioning in rats
}

\author{
ALAIN R. MARCHAND \\ Laboratoire d'Ethologie et Neurobiologie, Strasbourg, France
}

\begin{abstract}
The expression of cardiac responses to sequences of two sounds was studied in restrained rats following discriminative trace or delay conditioning. Stimuli paired with a tail shock 10 sec later (CS1) elicited conditioned bradycardia. Unpaired or neutral stimuli (CSO) elicited mostly tachycardia. Rats did not learn to suppress responding to nonreinforced sequences with an interval of 6 sec between sounds. Responses to the second stimulus were significantly augmented following a CS1 stimulus, but not following a CS0 stimulus. Real-time summation of simple responses provided a more complete and quantitative prediction of dual responses than did resetting or facilitation. These results extend the time range over which summation may be observed from less than $2 \mathrm{sec}$ to at least $16 \mathrm{sec}$. They appear to be inconsistent with models involving competition between unitary representations of stimuli in shortterm memory and suggest the existence of multiple stimulus traces with independent time courses.
\end{abstract}

It is commonly admitted that the activity evoked in a sensory system by a given stimulus can persist for some time after stimulus offset (Sakurai, 1990). Pavlovian conditioning, for instance, can occur not only when a conditioned stimulus (CS) and an unconditioned stimulus (US) are contiguous in time (delay conditioning), but also when a gap, the trace interval, separates the CS offset from the US onset (Gormezano \& Kehoe, 1981). To explain how this protocol (trace conditioning) allowed the development of a conditioned response (CR) to the CS, Pavlov (1927) first proposed that some persistent representation, or trace, of the stimulus became conditioned at the instant at which the US occurred. The trace hypothesis has been supported mostly by studies of the efficacy of conditioning with various CS-US intervals (Mackintosh, 1974). Reports of an optimal conditioning interval imply that the CS trace rises in intensity to a maximum some time after CS onset, then gradually decays (Gormezano, Kehoe, \& Marshall, 1983). However, the optimal conditioning interval appears to depend on the response system involved (Lennartz \& Weinberger, 1992).

The trace hypothesis has been extended by some authors to account not only for conditioning, but also for retrieval, during the test phase in which the CS is presented alone. Responses conditioned with distinct CS-US intervals usually show different topographies (Marchand \& Kamper, 2000). In particular, the peak of a CR often occurs near the

The author acknowledges valuable discussions with A. Ungerer and thanks V. LoLordo for comments on an earlier version of the manuscript. He is grateful to W. Wolf for constructing much of the equipment and to A. Klepper for animal care. Correspondence concerning this article should be addressed to A. R. Marchand, Laboratoire de Neurosciences Comportementales et Cognitives, UMR 7521, 12 rue Goethe, F-67000 Strasbourg, France (e-mail: marchand@ neurochem. u-strasbg.fr). instant of US presentation(Smith, 1968). To explain the time course of CRs, in several spectral models of Pavlovian conditioning, a variety of trace components with distinct time courses have been postulated. They become independently conditioned, and the CR is related at every instant to the compounded intensity of the CS traces (Buhusi \& Schmajuk, 1999; Kehoe, Horne, Macrae, \& Horne, 1993). The same traces would thus both determine the intensity of conditioning and directly control the expression of the CR.

If so, the time course of CRs might be used to predict when conditioning can occur. Moreover, the existence of independent CS traces may be tested by studying the interaction between stimuli occurring in temporal proximity, to see how they become integrated into a single response. According to spectral models, responses to individual trace elements may summate. Real-time summation should, strictly speaking, preserve the time course of each response. Another type of interaction may consist of resetting, corresponding to a response to the more recent stimulus only (Buhusi \& Meck, 2000; Prosser, 1995). This response may be altered by facilitation, inhibition, or contrast - that is, increases or decreases of the last response owing to the presence of a prior stimulus (McKenna, Weinberger, \& Diamond, 1989) or to the change of stimulus. Finally, compounding may lead to a pattern of response during sequences that is altogether different from that to isolated stimuli-for instance, because of generalization decrement (Pearce, 1987) - and occasion setting would be characterized by a reduction of responses to nonreinforced sequences after training (Holland, 1998). A detailed analysis of integrated responses is thus needed to distinguish between the various alternatives.

A much studied continuous index of response has been a conditioned eyeblink response in the rabbit (Gormezano et al., 1983), also termed nictitating membrane response, or NMR. Some characteristics of stimulus traces have 
been probed by testing this response with multiple stimuli, within a time range of a second or two. The dynamics of the NMR after conditioning has been studied with serially presented CSs or by changing the duration or spacing of stimuli (Desmond \& Moore, 1991; Kehoe et al., 1993; Kehoe \& Napier, 1991). In particular, eyeblink responses to serial pairs of previously conditioned sounds have been studied (Kehoe et al., 1993, Experiment 4). The results showed that each stimulus could evoke a response with a timing corresponding to the CS-US interval. Furthermore, as the interval between successive stimuli was increased, a double peak of response tended to be observed. This observation is consistent with a summation process, as in spectral models (Buhusi \& Schmajuk, 1999; Grossberg \& Schmajuk, 1989; Kehoe et al., 1993).

This real-time summation process contrasts with psychophysical models in humans that involve short and long sensory stores (Cowan, 1984). Short-term memory models postulate that a CR depends on an unitary representation of the CS, which interferes in short-term memory with the representation of other stimuli. In the field of animal learning, a related model, SOP, has been proposed (Wagner, 1981), according to which CSs compete to activate elements in a single US node of limited capacity. Although each CS is assumed to have an independent, sensory node, the overall time course of the CR is determined and limited by the activation in the US node.

A limitation of NMR studies is that the CR spans only short time intervals, but other preparations are available, mostly involving emotional conditioning, that are known to support long trace intervals (Kamin, 1965; Lennartz \& Weinberger, 1992). The time course of conditioned fear has, for instance, been probed with startle potentiationfrom a fraction of a second to about a minute (Davis, Schlesinger, $\&$ Sorenson, 1989). The temporal resolution of this method was, however, limited by the use of discrete startle probe stimuli, which are applied at only one instant of each trial.

The cardiac CR is available on a second-by-second basis for long periods of time, before and throughout training, as opposed to most measures of conditioning. No overt response or even decision process is required from the subject. Yet the use of heart rate as a measure of conditioned fear has been somewhat debated, and cardiac CRs have not been extensively investigated within a trace paradigm (Fitzgerald \& Martin, 1971; Fitzgerald \& Teyler, 1970; Marchand \& Kamper, 2000; Meredith \& Schneiderman, 1967).

In a recent study, Marchand and Kamper (2000) studied trace fear conditioning to brief auditory stimuli in restrained rats. They reported that distinct cardiac CR topographies could be observed, depending on the CS-US interval. They suggested that the topography of these responses is related to a central state of the animal, rather than to cardiovascular dynamics. Reports that conditioned heart rate and blood pressure changes are relatively independent (Li, Lawler, Randall, \& Brown, 1997) also argue against peripheral determinants. It should, then, be possible to alter the time course of the trace and, therefore, of the response, by presenting a second stimulus during the trace interval.

One study investigated cardiac responses to serial auditory stimuli in restrained rats (Fitzgerald \& Stainbrook, 1986). These authors used two stimuli: CS + , previously paired with the US and eliciting bradycardia, and CS-, previously unpaired with the US and eliciting tachycardia. They showed that a first stimulus CS - did not inhibit responding to CS+ presented $6 \mathrm{sec}$ later. Responses to the sequence presented a transition from tachycardia to bradycardia within the same trial. The second part of the response did not differ from the bradycardiac response to $\mathrm{CS}+$ alone, but the detailed topography of CRs was not explored.

In the present study, real-time summation or interaction during the presentation of sequences of two auditory stimuli, previously trained in a differential trace or a delay conditioning protocol, was examined. Three distinct sounds were used, one of which was not presented during initial discriminative training. This was intended to control for special (inhibitory) properties possibly acquired during discriminative training. Compound responses were compared with theoretical predictions and were found to be largely consistent with a summation account.

\section{METHOD}

\section{Subjects}

The animals were 24 experimentally naive male Long-Evans hooded rats (Rattus norvegicus) obtained from a commercial supply (Centre d'Elevage et de Recherche Janvier) at the age of 7 weeks. They were housed in groups of 4-5 in translucent plastic cages (width, $25 \mathrm{~cm}$; depth, $40 \mathrm{~cm}$; height, $18 \mathrm{~cm}$ ), with ad-lib access to food and water. The colony room was maintained on a 12:12-h light:dark cycle, and experimental sessions occurred during the light part of that cycle. The rats were 8-10 weeks old at the time of electrode implantation and weighed 245-335 g. They were then housed individually and, 4-20 days later, were handled for $3 \mathrm{~min}$ on each of 5 successive days. Implantation was performed without immobilization under clean, but not aseptic, conditions, using sodium pentobarbital $(65 \mathrm{mg} / \mathrm{kg}$ i.p.) anesthesia. Through a small incision in the upper right and lower left thorax, sterile electrodes (5-mm brass disk soldered to insulated wire) were sutured under the chest muscles. The wires were passed under the skin, using a hollow needle, to the back of the neck and were quickly soldered to a flying connector and then embedded in dental cement. The incisions were cleaned with antiseptics before closing. Care was taken throughout the experiment to minimize pain or discomfort of the animals.

\section{Apparatus}

The rats were restrained in one of four identical, opaque $20-\mathrm{cm}-$ long PVC boxes with holes (1-cm diameter), under an adjustable inverted U-shaped tube (7.5- $\mathrm{cm}$ diameter). The tail and leads to the connector were allowed out of the back of the box. Each restraining box was enclosed within one of four identical custom made soundattenuating chambers (width, $19 \mathrm{~cm}$; depth, $36 \mathrm{~cm}$; height, $22 \mathrm{~cm}$ ). A ventilation fan provided background noise $(65 \mathrm{~dB})$. Dim light was provided by a $12-\mathrm{V}, 40-\mathrm{mA}$ bulb mounted on the ceiling. The chamber contained an $8-\mathrm{cm}$ speaker mounted on the ceiling. The heart signal was amplified and bandpass filtered between 100 and $500 \mathrm{~Hz}$ by a WPI Iso-DAM8 amplifier (World Precision Instruments, Sarasota, FL), monitored with a digital oscilloscope, and recorded. It was simultaneously sampled on line through a CED-1401+ interface to 
a PC-type microcomputer using CED Spike2 software (Cambridge Electronic Design, Cambridge, England). The US was a tail shock from an A.M.P.I. Master-8 stimulator with Iso-Flex isolation units (A.M.P.I, Jerusalem, Israel), producing a 500-msec duration train of twenty-five 10-msec, $0.8-\mathrm{mA}$ pulses. The shock electrodes were two 1.1-cm stainless steel disks with shafts that could be secured along the tail with surgical adhesive tape. Electrical contact was ensured by a small drop of conductive paste. The auditory stimuli, generated from a 44-kHz digitized file, were $2000-\mathrm{Hz}$ tone (T) $80 \mathrm{dBa}$ in intensity, a train of six clicks (clicker, C) $75 \mathrm{dBa}$ in intensity, and white noise $(\mathrm{N}) 80 \mathrm{dBa}$ in intensity. The clicks were white noise exponentially damped with a 3-msec time constant.

\section{Procedure}

After the rats had recovered from surgery, they were made familiar with the restraint and conditioning apparatus for $15 \mathrm{~min}$ on the $1 \mathrm{st}$ day and then for $35 \mathrm{~min}$ on the 3 successive days. No stimulus was delivered during these sessions. Throughout the experiment, 4 animals were simultaneously tested and then left to socially interact for 3-5 min. Three groups of 4 rats were assigned to a trace protocol with one type of sound (T, C, or N; duration, $0.5 \mathrm{sec}$ ) as CS1, the next as $\mathrm{CS} 2$, and the third sound as CS3, in a counterbalanced manner. Three other groups were subjected to a delay protocol with similarly counterbalance $d$ sounds, but of longer duration $(10.5 \mathrm{sec})$ so that the sound and the shock coterminated. Figure 1A depicts the time line of a session. The interval between sound onset and shock was $10 \mathrm{sec}$ in all cases. Discriminative conditioning was conducted for 10 daily sessions, to control for possible nonassociative processes. On each session, four reinforced trials (CS1-US) were randomly interspersed with four CS2-alone trials (intertrial interval [ITI], $270 \pm 150 \mathrm{sec}$ ). The first stimulus was delivered after $7 \mathrm{~min}$ in the conditioning chamber (Supple \& Leaton, 1990). The third sound, CS3, was never presented at this stage.

Two additional sessions (11 and 12) were then conducted, during which the novel CS3 was introduced and only half the CS1 trials were reinforced. Their purpose was to explore the whole response to CS1 and to habituate orienting responses to CS3. These sessions each comprised 16 trials: 4 CS 1-US, 4 CS 1 alone, 4 CS2-alone, and $4 \mathrm{CS} 3$-alone trials (ITI, $135 \pm 75 \mathrm{sec}$ ).

Finally, 14 test sessions (Sessions 13-26) were conducted daily, using all serial combinations of tone, clicker, or noise. Although CS1 was embedded within some sequences, they were never reinforced (Figure 1B). Because of the number of trials required, it was not possible to conduct this testing in extinction. Each session comprised 28 stimuli ( 24 in the first 4 sessions) randomly interspersed without repetitions: two reinforced CS1 presentations (then increased to six to prevent extinction, since responses were rather weak), plus two nonreinforced presentations of CS2, CS3, and each of nine possible sequences of two sounds (ITI, $90 \pm 45 \mathrm{sec}$ ). The second sound was presented $6 \mathrm{sec}$ after the onset of the first sound. In the delay group,

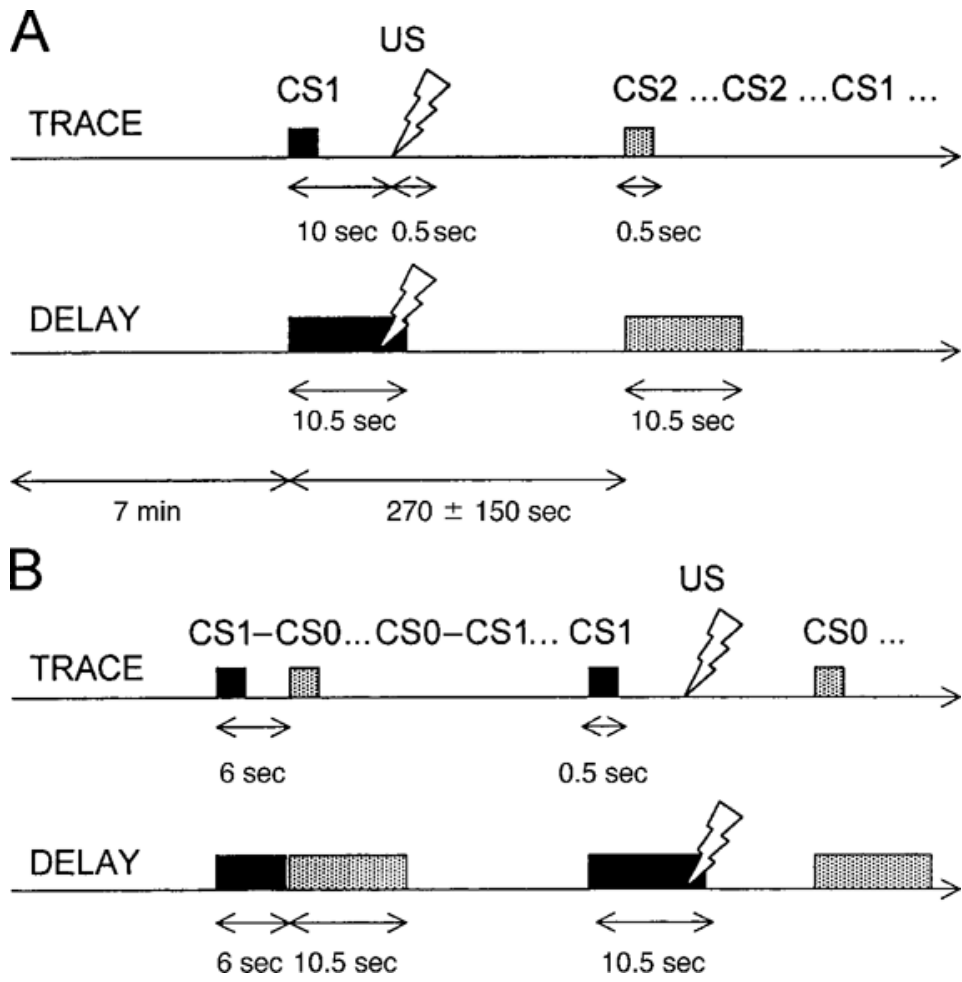

Figure 1. Time line of conditioning and test sessions. Stimuli were $0.5 \mathrm{sec}$ long in group trace and preceded the shock unconditioned stimulus (US) by 10 sec. In group delay, the conditioned stimulus (CS) was $10.5 \mathrm{sec}$ long and coterminated with the 0.5 -sec shock US. (A) Conditioning sessions: four reinforced CS1s and four nonreinforced CS2s were presented in pseudorandom order. (B) Test sessions: All possible simple and dual stimuli were presented in pseudorandom order. Simple CS1s were reinforced. The onsets of sounds in dual stimuli were separated by 6 sec. In group delay, the second stimulus replaced the first one after 6 sec. 
the first sound was cut off after $6 \mathrm{sec}$, partly for technical reasons and partly because overlapping sounds would constitute a novel event for the rat. The second sound started $250 \mathrm{msec}$ later and lasted $10.5 \mathrm{sec}$.

\section{Data Analysis}

The sampled heart voltage signals were submitted to a digital threshold procedure to detect the R-wave of the cardiogram, using a CED Spike2 program, and then were visually inspected to remove muscle artifacts. Sounds, shocks, and beat times were stored on disk and were processed using custom programs. The interbeat intervals (IBIs) were collected into 10 prestimulus and 30 poststimulus bins of $1 \mathrm{sec}$, based on when the end of the IBI occurred. Within each bin, only the average period was analyzed (see Berntson, Cacioppo, \& Quigley, 1995), although the times of individual beats were not discarded. All data were computed as the IBI change with respect to a 10 -sec pretrial baseline. Bin data for every trial, session, and subject were imported into Microsoft EXCEL for further analysis and display.

Conditioning sessions (1-10) and test sessions (13-26) were analyzed separately because of their different designs. Sessions 11 and 12 were not included in the analysis. Repeated measures analyses of variance (ANOVAs) were performed with STATISTICA software. The Student-Newman-Keuls (SNK) post hoc test was then used to inspect significant effects. The probability threshold for rejection of the null hypothesis was .05 throughout.

In the conditioning phase, only the magnitudes of cardiac responses to CS1 and CS2 were compared. The mean period change was measured on the 10th second after sound onset (Marchand \& Kamper, 2000). For robustness, all data were collapsed with respect to the physical nature of the stimuli (tone, clicker, or noise, counterbalanced). Response amplitudes were compared over successive blocks of two sessions. The design involved three factors: The between-subjects factor was protocol, with group trace (short CS) and group delay (long CS). Repeated measures were reinforcement (CS1 or CS2) and session (blocks).

For the test phase, the data were pooled over successive blocks (weeks) of four, five, and five sessions, respectively, and the topography of the CRs following the last stimulus was detailed instant by instant for $10 \mathrm{sec}$. Since this analysis involved many trial types (12) and because very little difference was found between the effects of $\mathrm{CS} 2$ and CS3, these two stimuli were collapsed and were termed CS0. In this ANOVA, the effects of protocol were ignored, so there were three factors: week, instant, and trial type. Subsequently, the values in each bin were collapsed across all test sessions.

The effect of the sequence on the response to the second stimulus was evaluated: Response was evaluated near its peak-namely, $10 \mathrm{sec}$ after the onset of the second stimulus in compound trials (16th second) and $10 \mathrm{sec}$ after the only stimulus in simple trials. The analysis was repeated at the expected time of the first response peak, $6 \mathrm{sec}$ earlier. This ANOVA, followed by SNK post hoc tests, involved one between-subjects factor (protocol) and one repeated measure factor (trial type).

\section{Criteria for Response Interaction}

Compound response amplitude was the first criterion by which to evaluate the possible modes of interaction between successive responses (Table 1). For this analysis, the responses to the last stimulus alone were aligned to start $6 \mathrm{sec}$ after the onset of dual trials. Whereas inhibition or other types of interaction are expected to decrease response amplitudes with respect to those to the last stimulus alone, a simple resetting process should leave them unchanged. Contrast and facilitation may be distinguished according to trial type. Increased responses may indicate facilitation if they occur even with repetitions of the same stimulus or contrast if trials involving only two different stimuli are concerned. Finally, response amplitude should also be increased by summation, and in a quantitatively specified manner.

Actually, the second criterion was whether observed peak amplitudes of responses significantly deviated from predicted real-time summation. A separate ANOVA directly tested this hypothesis, the factors being protocol, compound trial type, and summation (observed vs. predicted). In theory, summation consists of adding two simple responses, aligned to correspond to the actual time of delivery of each stimulus. So a prediction for the second peak was computed as the sum of the 16th second of response to the first stimulus alone and the 10th second of response to the second stimulus alone. For the first peak, the sum applied to the 10th and 4th seconds, respectively. It was sometimes necessary to extrapolate responses to $\mathrm{CS} 1$ beyond the time of shock. An estimate of this late CR 1 response was provided by the tail of response in CS0-CS1 trials, slightly scaled down (see Figure 4).

Even when no significant deviation from the summation hypothesis could be observed, it was important to show that the response was not simply facilitated but that both stimuli controlled the dynamics of the responses. So a correlation analysis was used as a third criterion (Table 2). The observed dual responses were correlated, on the one hand, with the response to the second stimulus alone, appropriately timed, and on the other hand, with the theoretical summation response in the same subject. Both should show a positive correlation whether summation or resetting/facilitation occurred, because the most recent stimulus is expected to significantly contribute to the compound response. However, if summation occurred, the correlation with the sum should be higher than that with the second stimulus alone, because the prediction would be improved by the addition of the first stimulus. This is indicated by + when a weaker positive correlation is expected and by ++ when a stronger positive correlation is expected. Conversely, if responses to the second stimulus were simply reset or facilitated, the correlation with the sum should be lower than that with the second stimulus, because of the irrelevant contribution of the first stimulus. A further advantage of the correlation approach is that it relies on relative amplitudes, rather than on absolute amplitudes. So, provided facilitation is consistent between subjects, testing the facilitation hypothesis is just the same as testing the resetting hypothesis. Contrast leads to a prediction similar to that for facilitation, because only the response to the last stimulus is relevant. Other modes of interaction do not lead to clear-cut predictions about correlation. Correlation coefficients were computed for each sequence-namely, CS1-CS1, CS0-CS1, CS1-CS0, and CS0-CS0. Only the 16th and 10th seconds of dual trials were considered, as before. The Bonferroni corrected error rate for eight comparisons was 0.0063 . So, with 24 subjects, correlation coeff icients higher than .542 were significant. Fisher's $z$ transform (Edwards, 1976) was used to compare correlation coefficients corresponding to the summation hypothesis and those corresponding to the facilitation/resetting hypothesis.

\section{RESULTS}

\section{Discriminative Conditioning}

The unconditioned response to shock was a biphasic, prolonged cardioacceleration (not shown). Temporary in-

Table 1

Amplitude Criterion for Response Interaction

\begin{tabular}{|c|c|c|c|c|c|}
\hline Trial Type & Resetting & Contrast & Facilitation & Summation & Other \\
\hline $\mathrm{CSO}$ & $=\mathrm{CR} 0$ & $=\mathrm{CR} 0$ & $>\mathrm{CR} 0$ & $>\mathrm{CR} 0$ & $<\mathrm{CR} 0$ \\
\hline $\mathrm{CSO}$ & $=\mathrm{CR} 1$ & $>\mathrm{CR} 1$ & 1 & $>\mathrm{CR} 1$ & $<\mathrm{CR} 1$ \\
\hline CS $1-\mathrm{CS} 0$ & $=\mathrm{CR} 0$ & $>\mathrm{CR} 0$ & $>\mathrm{CR} 0$ & $>\mathrm{CR} 0$ & $<\mathrm{CR} 0$ \\
\hline CS1-CS 1 & $=\mathrm{CR} 1$ & $=\mathrm{CR} 1$ & $>\mathrm{CR} 1$ & $>\mathrm{CR} 1$ & $<\mathrm{CR} 1$ \\
\hline
\end{tabular}


Table 2

Correlation Criterion for Response Interaction

\begin{tabular}{lccccc}
\hline Prediction & Resetting & Contrast & Facilitation & Summation & Other \\
\hline Last & ++ & ++ & ++ & + & $?$ \\
First + last & + & + & + & ++ & $?$ \\
\hline
\end{tabular}

creases in the baseline heart period were observed in both groups between the last familiarization session $(138 \mathrm{msec})$ and the first conditioning session $(152 \mathrm{msec}$; data not shown). During the test phase, significant baseline fluctuations persisted $[F(1,6)=2.38]$, but no consistent trend was observed.

Figure 2 depicts the evolution of CRs as a function of successive blocks of two sessions of conditioning and test. CRs to CS1 or CS2 in restrained animals were bradycardiac (Marchand \& Kamper, 2000; Supple \& Leaton, 1990), and the level of conditioning was independent of the conditioning protocol (trace or delay). In both groups, responding dropped markedly at the onset of test sessions (Sessions 13 and later) but then remained approximately stable. The magnitude of the CRs did not correlate with variations in the pretrial baseline over test sessions $(r<.23)$.

The initial bradycardiac responses to the sounds were not discriminative (Marchand \& Kamper, 2000). However, discrimination between CS1 and CS2 rapidly developed. A three-factors repeated measures ANOVA on responses at instant $10 \mathrm{sec}$ and successive blocks of two conditioning sessions was performed. An effect of reinforcement $[F(1,22)=77.6]$ showed that the animals discriminated between stimuli and responded more to the re- inforced CS1 than to CS2. There was an effect of sessions $[F(4,88)=21.8]$, and reinforcement and session interacted $[F(4,72)=7.16]$, meaning that discrimination developed gradually. The SNK test indicated that discrimination appeared by Sessions 3-4 with delay conditioning and by Sessions 5-6 with trace conditioning.

Protocol had no main effect $[F(1,22)=0.42]$ and did not interact with reinforcement $[F(1,22)=1.21]$. But a protocol $\times$ session interaction $[F(4,72)=4$.67] showed that the protocol (or stimulus duration) significantly influenced the rate of conditioning. The SNK test indicated that the delay group tended to respond more on Sessions 1-2 and showed an earlier decline in response on Sessions 5-6. No three-way interaction was found $[F(4,88)=1.75]$. On the whole, the trace protocol slightly retarded simple conditioning and discriminative responding.

\section{Response Topography After Conditioning}

During the next two sessions (11 and 12), CS1 was shifted from total to partial reinforcement (50\%), to evaluate CR1 topography over the whole $30-$ sec interval. Figure 3 shows, on nonreinforced test trials, the average topography of conditioned responses to CS1 and to CS2 for the trace group (short CS) and the delay group (long CS). In the trace group, the bradycardiac response CS1 peaked on the 9th second, then decayed smoothly after shock omission. In the delay group, the response increased until the 10th second. At CS1 offset, a cardioacceleration superimposed on the ongoing response. The nonreinforced sound, CS2, elicited a tachycardiac episode (cardioacceleration), followed by a very weak bradycardiac response. The delay

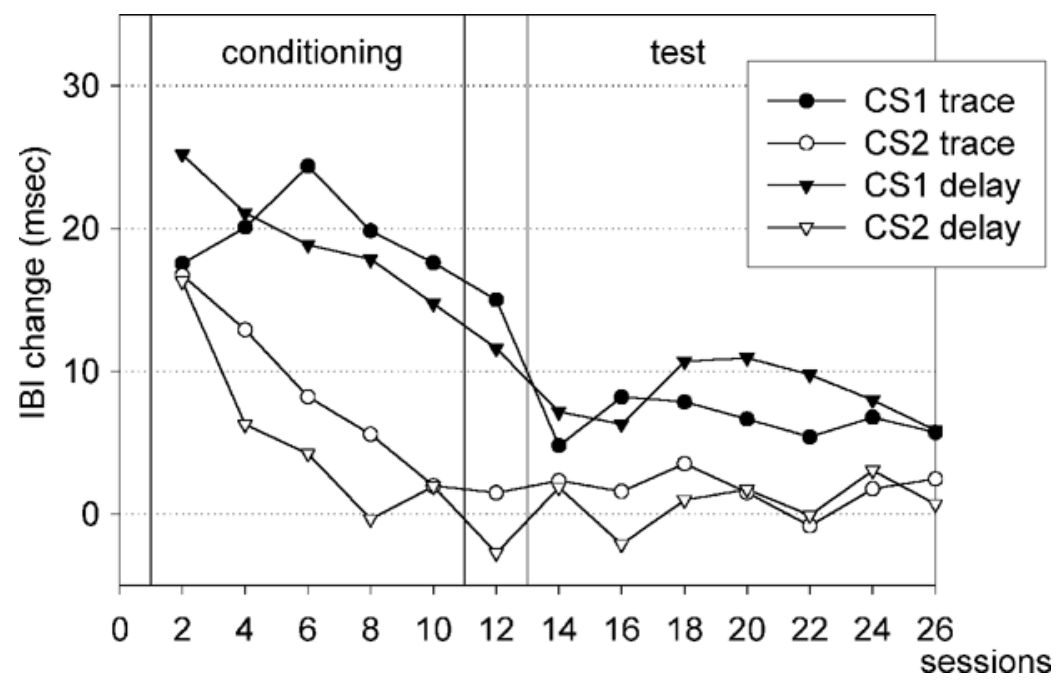

Figure 2. Discrim inative trace and delay conditioning: evolution of the response on the 10th second to the reinforced (CS1) and nonreinforced (CS2) sounds as a function of blocks of two training sessions. Interbeat interval (IBI) changes with respect to pretrial baseline are plotted for group trace and group delay. Bradycardia is upward. Responses to tones, clickers, or noises were pooled together, only their reinforcement status being considered. 


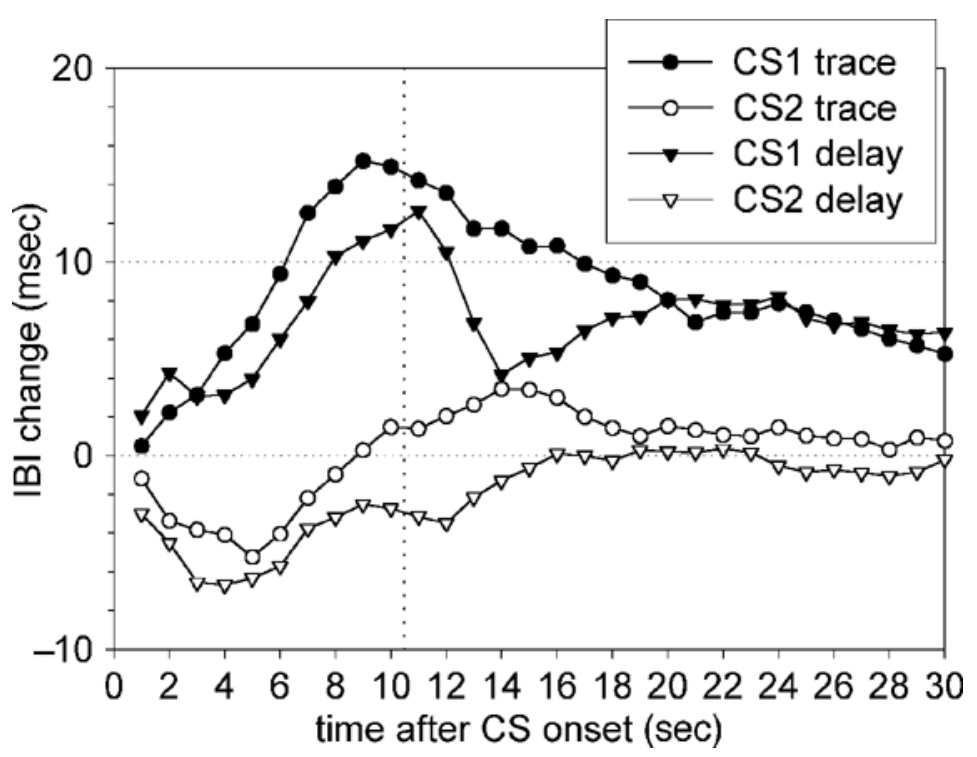

Figure 3. Topography of responses to simple conditioned stimuli CS1 and CS2 during Sessions 11-12 collapsed, comparing groups trace and delay. Interbeat interval (IBI) changes computed for each second were plotted as a function of the time after CS onset. For CS1, only test trials in which the US was omitted are represented. Responses to $\mathrm{CS3}$ are not shown, since they were rapidly habituating during this period. The vertical dotted line corresponds to the instant of sound offset in the delay group, as well as of omitted shock in both groups.

group responded to CS2 with a longer tachycardia and, on average, no bradycardia.

The novel sound CS3, on its first presentation, elicited a transient bradycardiac orienting response (Graham \& Clifton, 1966), which rapidly decayed to tachycardia after four presentations. During the subsequent test phase, individual responses to CS2, CS3, or both showed weak bradycardia in half of the rats $(3-8 \mathrm{msec}$ period change) and, in the other rats, only tachycardia. Compound responses involving CS2 or CS3 were also similar during the test phase, except on the 10th second, where responses following CS2 as a first stimulus were larger than those following CS3. In order to avoid complexity, data involving CS2 and CS3 are averaged and designated CSO (stimulus) or CR0 (response). Furthermore, when possible, analyses are presented with trace and delay groups pooled together.

\section{Effects of Training During the Test Phase}

During the test phase, it was important to determine whether the rats learned that sequences were never reinforced, since this would defeat the goal of the study. Test sessions were pooled into 3 weeks (Sessions 13-16, 17-21, and 22-26). The topography of response to sequences was analyzed at each instant from the 7th to the 16th second in dual trials. Single trials were similarly analyzed from the 1 st to the 10th second. Shock occurrence precluded a longer analysis of CR1.

The data were subjected to a three-way ANOVA with trial type, instant, and weeks as repeated measures. Trial type and instant had significant main effects and interacted (all $F_{\mathrm{S}}>13.3$ ), which called for further analysis (see below). Week had no main effect $[F(2,46)=0.69]$ and did not interact with trial type $[F(10,230)=0.60]$. Nevertheless, a significant week $X$ instant interaction was found $[F(18,414)=4.71]$, as well as a three-way type $\times$ week $\times$ instant interaction $[F(90,2070)=1.31]$. Inspection of the effect showed that it resulted mainly from a larger response during Week 2 with some trial types, whereas no decrease occurred from Week 1 to Week 3. Factor instant was involved, because responses were initiated from a similar baseline, but response topography was otherwise unchanged across weeks. It was thus clear that during these test sessions, the rats did not learn not to respond to sequences. The data were subsequently collapsed over all test sessions.

\section{Topography of Dual Responses}

The average response topography for each trial type and each group can be seen in Figure 4, along with simulations of the summation and resetting hypotheses (see the Method section). During the first $6 \mathrm{sec}$, only the response evoked by the first stimulus can appear.

An inspection of Figure 4 shows that the time course of a dual response after the 6th second was characterized by a rapid rise and a slow decay. It either matched or roughly paralleled a simple response to the second, more recent stimulus alone, which was the prediction for resetting. Following a first CSO stimulus (upper panels), actual responses 

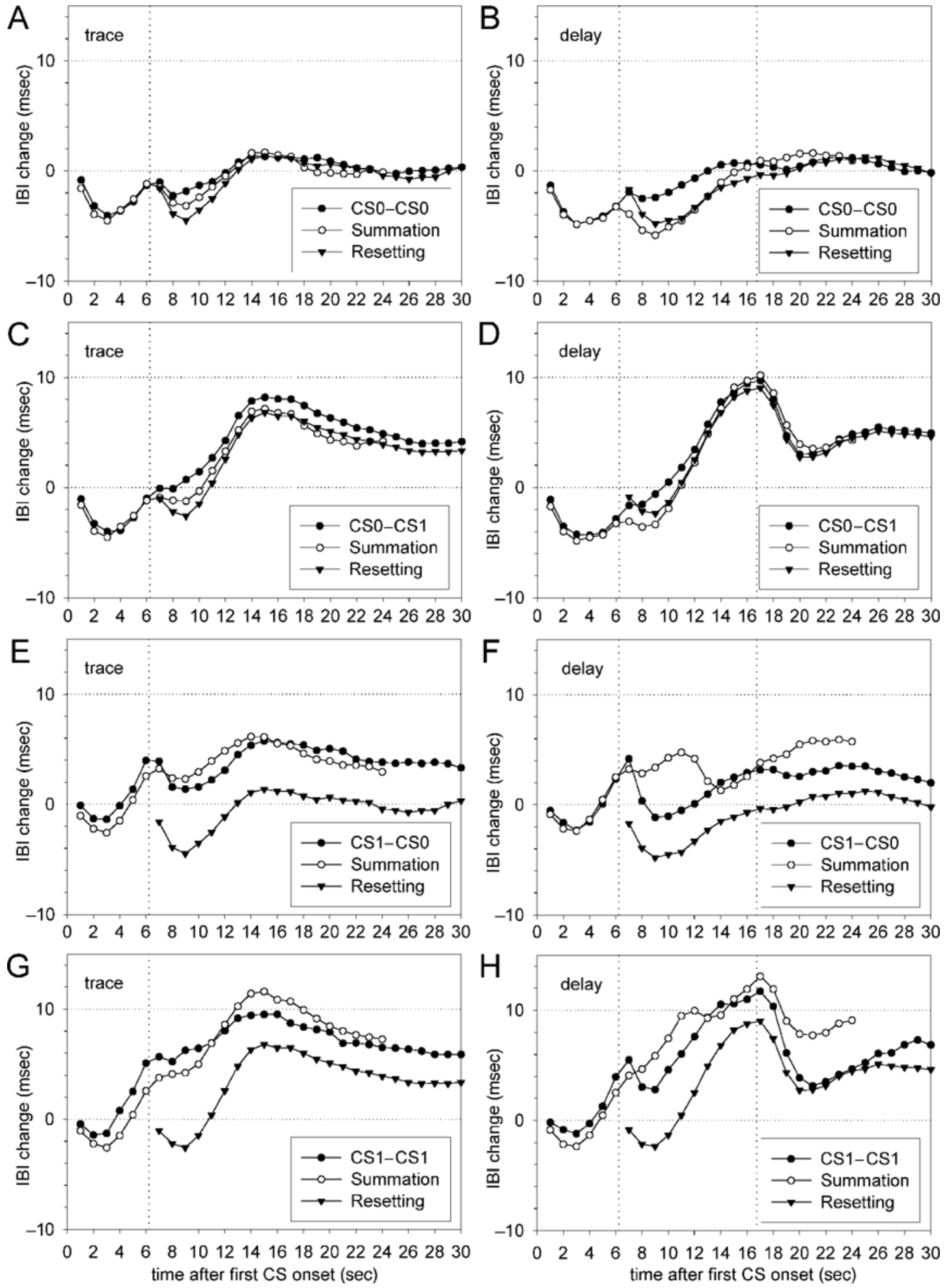

Figure 4. Topography of responses to dual stimuli. Representation as in Figure 3. Each pair of figures (A-B to G-H) corresponds to a trial type. Left panels, group trace; right panels, group delay. Observed responses, collapsed across all Test Sessions 13-26, a re shown, along with theoretical responses corresponding to summation or resetting. CS0 represents CS2 and CS3 pooled together. Summation: second-by-second addition of the simple responses to the first stimulus and to the last stimulus, appropriately delayed. Resetting: simple response to the last stimulus CS1 or CS0, delayed to correspond to its actual onset (first vertical dotted line, $6.25 \mathrm{sec}$ ). The second vertical line $(16.75 \mathrm{sec})$ in the delay group indicates offset of the second sound. 
and responses predicted by either summation or resetting were very much the same, except for their initial tachycardiac episode, which seemed weaker than predicted. On the other hand, responses following a first CS1 stimulus (lower panels) were markedly enhanced with respect to the prediction for resetting. The observed dual responses were better predicted by summation.

In the trace group, the average response peaked by the 15 th second - that is, $9 \mathrm{sec}$ after the onset of the second stimulus (Figures 4A, 4C, 4E, and 4G). So the second stimulus in a sequence evoked a response with a distinctive timing, which was consistent with resetting or summation. In the delay group, responses to sequences ending with CS1 (Figures 4D and 4H) also peaked with a timing appropriate to the second stimulus (16th second). For CSOCSO and CS1-CS0 sequences, responses were weak, and their timing difficult to evaluate (Figures $4 \mathrm{~B}$ and $4 \mathrm{~F}$ ).

Figure 4 also shows that the peak of the first response, expected on the 9th or 10th second, was generally masked by a tachycardiac episode occurring at the onset of the second stimulus. Whether or not the first response was interrupted was not clear. In the delay group, a large tachycardia was also present at the offset of the second CS1 (Figures 4D and 4H, dotted line). Bradycardia generally resumed after tachycardiac episodes, which suggests that tachycardia simply summated on top of bradycardiac responses. Enhanced responses to the second stimulus in a sequence could indicate either a summation or a facilitation effect. Specific analyses of the interaction between trial type and instantaneous response were targeted at the 16th and 10th seconds in order to try to clarify this issue.

\section{Responses to the Last Stimulus in a Sequence}

The 16th second of dual trials approximately corresponds to the peak response to the second sound. It was compared with the peak (10th second) of simple trials in a repeated measures ANOVA with two factors, protocol and trial type. Protocol had no main effect $[F(1,22)=0.01]$. Trial type showed an effect $[F(5,110)=47.8]$ and interacted with protocol $[F(5,110)=2.53]$. Post hoc tests indicated that responses to CS1-CS0 sequences were more pronounced in the trace group (Figures 4E and 4F), and a few differences attained significance only in one group or the other. The most recent stimulus was a major determinant of response amplitude, since sequences ending with CS1 elicited stronger responses than did sequences ending with CSO.

Responses to various trial types all differed significantly, except CSO-CSO versus CSO, and CSO-CS1 versus either CS1 or CS1-CS1. So, a prior nonreinforced stimulus CS0 had no effect on the amplitude of subsequent responses. CS1-CS1 and CS1-CS0 responses were significantly higher than CR1 or CR0, respectively. Thus, a prior CS1, but not CS0, enhanced responding to any subsequent stimulus. This indicates a deviation from the resetting hypothesis, but facilitation could still account for this effect.
Compound responses were compared with theoretical summation responses by means of a three-way repeated measures ANOVA. Protocol was the between-subjects factor, and trial type (compound trials only) and summation (observed vs. theoretical) were within-subjects factors. Significant effects concerned trial type and its interaction with protocol $[F(3,66)=52.8$ and $F(3,66)=3.42]$, but no significant effect or interaction was detected involving summation (largest $F=1.59$ ). Thus, compound response amplitudes did not significantly deviate from what summation predicted.

To further distinguish between summation and facilitation, compound responses were correlated across subjects with the predicted summation response or the predicted facilitation/resetting response (see the Method section). Table 3 (right part) shows the correlation coefficients for each trial type at the time of the second peak. All coefficients in this part of the table were above .542 and, therefore, significant. Responses to repeated stimuli, such as $\mathrm{CS} 0-\mathrm{CS} 0$ and CS1-CS1, tended to correlate slightly better with the predicted facilitation/resetting, as did CSO$\mathrm{CS} 1$. On the other hand, with CS1-CS0, the correlation with the predicted facilitated response appeared weaker than that with summation. None of these differences in correlation between the facilitation and the summation hypotheses was significant, however. Thus, the correlation analysis on the 16th second did not lead to a definite conclusion, being consistent with either facilitation or summation.

\section{Responses to the First Stimulus}

It could be argued that the effect of the first stimulus should be best seen near its peak, about the 10th second, since a summation account predicts that the initial response to the second stimulus should simply superimpose on the ongoing first response. So, dual responses were examined $4 \mathrm{sec}$ after the last stimulus, in order to detect deviations from resetting or summation. The amplitudes of responses on the 10th second of dual trials and the corresponding 4th second of simple trials were submitted to a repeated measures ANOVA. Factors were trial type and protocol. Trial type showed an effect $[F(5,110)=30.1]$, but not protocol $[F(1,22)=2.23]$, and no interaction emerged $[F(5,110)=0.48]$. Responses to various trial types differed significantly, except CS1-CS0 versus either CSO$\mathrm{CS} 0$ or CSO-CS1 and CS0-CS0 versus CS1. Systematic deviations from resetting were observed, since CS1-CS1

Table 3

Correlation Between Observed and Predicted Responses

\begin{tabular}{lccccc}
\hline & \multicolumn{2}{c}{ Peak 1 } & & \multicolumn{2}{c}{ Peak 2 } \\
\cline { 2 - 3 } \cline { 5 - 6 } Trial Type & Last & First + Last & & Last & First + Last \\
\hline CS0-CS0 & .65 & .67 & & .61 & .56 \\
CS0-CS1 & .88 & .85 & & .87 & .77 \\
CS1-CS0 & .23 & .53 & & .60 & .77 \\
CS1-CS1 & .44 & .53 & & .80 & .79 \\
\hline
\end{tabular}


and $\mathrm{CS} 0-\mathrm{CS} 1$ responses were significantly higher than $\mathrm{CR} 1$, whereas CS1-CS0 and CS0-CS0 were higher than CR0. So, any prior stimulus enhanced responding on the 10 th second.

Compound responses were again compared with predicted summation responses in an ANOVA. The only main effect was that of trial type $[F(3,66)=32.6]$. A significant interaction involved summation and trial type $[F(3,66)=$ $12.2]$, and a three-way interaction was also found $[F(3,66)=$ 4.56]. Other effects were not significant (largest $F=1.23$ ). The SNK test revealed no significant difference with summation in the trace group, but a significant deviation from summation with all trial types in the delay group. Nevertheless, a summation account appeared to predict compound response amplitudes in the trace group.

The data were again submitted to the correlation analysis. Table 3 (left part) shows correlation coefficients for each trial type at the expected time for the first peak. In this part of the table, only trials starting with CSO yielded significant correlation coefficients. Both for CSO-CSO and CS0-CS1, the correlation with the predicted facilitation/ resetting was quite similar to that for summation, and no significant difference emerged. So, on the 10th second, when the influence of the first stimulus should be maximal, the prediction made by the summation account was no better than that made on the basis of the second stimulus alone.

Finally, inspection of individual CS1-CS0 response curves showed some heterogeneity in dual responses. Very weak responses in 6 rats made the analysis difficult. In 6 delay rats and 2 trace rats, there was some evidence of resetting when a first, large response disappeared after the second stimulus.

\section{DISCUSSION}

\section{Evidence for Response Summation}

The present study describes the interaction between previously conditioned auditory traces. Its aim was to test a trace summation hypothesis, according to which responses could be simultaneously under the control of both stimuli. This hypothesis states that a continued response to a first stimulus should be detectable during a response to a second stimulus. In the present experiments, there was no evidence that responses to sequences declined during 14 test sessions, as if the rats had not learned that sequences were never reinforced. The compound responses did not appear to evolve, so it may be assumed that the pattern of integration of dual stimuli was present from the start. Responses in sequences never appeared diminished with respect to the same responses in isolation, which excludes both inhibition and generalization decrement.

Thus, our results are consistent with those of Fitzgerald and Stainbrook (1986). In trials very similar to CS2-CS1 trials in our delay group, they found no effect of the putative conditioned inhibitor CS2 on the following excitatory response to CS1. So the second auditory stimulus was processed without inhibitory summation from the preced- ing one. Although this is contrary to what would be expected if CS2 possessed conditioned inhibitory properties (Mackintosh, 1974, pp. 33-34), the possibility remains, in both experiments, that different results might have been observed with specific inhibitory training procedures, such as a Pavlovian conditioned inhibition procedure (Yin, Barnet, \& Miller, 1994).

On the contrary, in the present study, one had to deal with an augmented second CR in most cases. After a first CSO stimulus, this weak augmentation was significant on the 10 th second but did not extend until the 16th second. After a first CS1 stimulus, the augmentation was quite prominent, and it was prolonged in CS1-CSO sequences. The results do not provide any evidence of occasion setting, compounding, or inhibition.

Two types of compound responses ( $\mathrm{CSO}-\mathrm{CS} 0$ and $\mathrm{CSO}$ CS1) did not differ from responses to the second stimulus alone on the 16th second but could just as well be predicted by summation as by a simple resetting effect. The weak response to the first CSO stimulus probably precluded any detection of summation. In their early part (10th second), the same responses were nevertheless enhanced with respect to the second stimulus alone. This enhancement should not be attributed to a contrast effect, since both responses to CSO-CS1 (contrast) and CSOCSO responses (no contrast) were enhanced with respect to CR1 and CR0, respectively. Deviations from summation were detected only in the delay group.

These observations indicate that a prior neutral stimulus (CS0) induces a weak response that appears to persist and summate only shortly after the second stimulus occurs.

Two other trial types showed significantly enhanced responses on the 16th second (CS1-CS0 and CS1-CS1). In this case, the response amplitudes were well predicted by real-time summation (Figure 4). This conclusion relies on within-subjects comparisons, which were sensitive enough to detect small differences with respect to responses to the second stimulus alone (e.g., after CS0 on the 10th second). The correlation analysis did not favor either summation or facilitation. On the other hand, facilitation would involve an arbitrary gain parameter. In particular, no facilitation at all occurred after a CSO stimulus (16th second). However, the summation hypothesis accurately predicted compound response amplitudes both after CSO (no increase in response) and after CS1 (increased responses). On the 10th second, the pattern of results was quite similar, except for weaker correlation coefficients and, in the delay group, a significant deviation from summation. Since the first sound was cut off after $6 \mathrm{sec}$ in dual trials with the delay group, deviations from summation probably reflect the fact that the first response, based on a stimulus $10.5 \mathrm{sec}$ long, was overestimated. In contrast, summation was preserved in the trace group.

In summary, the data may be best described as follows: The response to a prior $\mathrm{CS} 1$ - the stimulus associated with shock-summated with that to the subsequent stimulus, even when the second response was at its peak. 


\section{Stimulus Traces and Response Systems}

There may have been some heterogeneity in the way the rats integrated responses to successive stimuli, and the weakness of some individual responses was a handicap in the analysis. In further studies, partial reinforcement of CS1 during conditioning might be used to avoid extinction between the conditioning and the test phases. In addition, test trials with CS1 alone would provide the whole time course of CR1.

It was expected, among other possibilities, that when CSO followed CS1, the initial strong CR1 might be suppressed, according to a limited capacity hypothesis (resetting/ facilitation). There was no strong evidence that this occurred, and, on average, responses to CS1-CS0 sequences were quite pronounced. Although neutral stimuli yielded quite small amplitudes of response and summation was not always clearly separable from facilitation, real-time summation provided both a simple and a quantitative account of all the results.

These results are consistent with those obtained in the NMR preparation in general (Desmond \& Moore, 1991), and they extend the time range over which summation may be observed. Double responses in the NMR preparation have been taken as evidence in favor of spectral models, where multiple trace elements can become independently conditioned and can summate in responding. In particular, double responses to pairs of previously conditioned sounds have been observed (Kehoe et al., 1993, Experiment 4 ). We might have attempted, in our experiments, to manipulate the interval of time separating the stimuli within a sequence. Actually, a similar pattern of results was observed in a distinct experiment (not included here because of differences in design), in which the two stimuli were separated by an interval of $4 \mathrm{sec}$ instead of $6 \mathrm{sec}$. However, the cardiac response does not show a well-defined peak. Usually, only the second peak of response could be observed in the topography of compound responses, at a time at which the first response was slowly decaying. So it is doubtful that double peaks could be reliably identified.

Real-time summation implies that a specific representation of each stimulus (trace) is held in parallel in memory at every instant, as in spectral models. In human models of auditory memory (Cowan, 1984), the shorter store (up to $350 \mathrm{msec}$ ) is incompatible with summation, since each stimulus overwrites the previous one. The longer store (up to $20 \mathrm{sec}$ ) can hold more than one stimulus but is characterized by partial interference altering the response to earlier stimuli. In animal studies, Wagner's SOP model (1981) and its extension AESOP (Wagner \& Brandon, 1989) allow for partial summation, provided each CS activates only a fraction of the available US units (Wagner, 1981, pp. 35-36). These models also predict that more summation should occur with different CSs than with a repeated CS, but the present experiments were not intended to test this prediction. The present study nevertheless demonstrates that the integration of stimulus traces can be usefully explored through continuous monitoring of cardiac CRs.

Although AESOP suggests different neural substrates for NMRs (sensory nodes) and longer, fear-related responses (emotional), such as cardiac CR (Lennartz \& Weinberger, 1992; McNish, Betts, Brandon, \& Wagner, 1997; Vandercar \& Schneiderman, 1967), the present results suggest that real-time summation may be observed in both types of responses and on a longer time scale than with the NMR.

\section{REFERENCES}

Berntson, G. G., Cacioppo, J. T., \& Quigley, K. S. (1995). The metrics of cardiac chronotropism: Biometric perspectives. Psychophysiology, 32, 162-171.

Buhusi, C. V., \& Meck, W. H. (2000). Timing for the absence of a stimulus: The gap paradigm reversed. Journal of Experimental Psychology: Animal Behavior Processes, 26, 305-322.

Buhusi, C. V., \& Schmajuk, N. A. (1999). Timing in simple conditioning and occasion setting: A neural network approach. Behavioural Processes, 45, 33-57.

Cowan, N. (1984). On short and long auditory stores. Psychological Bulletin, 96, 341-370.

Davis, M., Schlesinger, L. S., \& Sorenson, C. A. (1989). Temporal specificity of fear conditioning: Effect of different conditioned stimulus-unconditioned stimulus intervals on the fear-potentiated startle effect. Journal of Experimental Psychology: Animal Behavior Processes, 15, 295-310.

Desmond, J. E., \& Moore, J. W. (1991). Altering the synchrony of stimulus trace processes: Tests of a neural-network model. Biological $\mathrm{Cy}$ bernetics, 65, 161-169.

EDWARDS, A. L. (1976). An introduction to linear regression and correlation. San Francisco: Freeman.

Fitzgerald, R. D., \& Martin, G. K. (1971). Heart-rate conditioning in rats as a function of interstimulus interval. Psychological Reports, 29, 1103-1110.

Fitzgerald, R. D., \& Stainbrook, G. L. (1986). Opposing heart rate reactions associated with behavioral states of excitation and inhibition. Physiology \& Behavior, 38, 5-9.

Fitzgerald, R. D., \& Tey Ler, T. J. (1970). Trace and delayed heart-rate conditioning in rats as a function of US intensity. Journal of Comparative \& Physiological Psychology, 70, 242-253.

Gormezano, I., \& Kehoe, E. J. (1981). Classical conditioning and the law of contiguity. In P. Harzem \& M. D. Zeiler (Eds.), Predictability, correlation and contiguity (pp. 1-45). New York: Wiley.

Gormezano, I., Kehoe, E. J., \& Marshall, B. S. (1983). Twenty years of classical conditioning research with the rabbit. Progress in Psychobiology \& Physiological Psychology, 10, 197-275.

Graham, F. K., \& Clifton, R. K. (1966). Heart-rate change as a component of the orienting response. Psychological Bulletin, 65, 305-320. Grossberg, S., \& Schmajuk, N. A. (1989). Neural dynamics of adaptive timing and temporal discrimination during associative learning. Neural Networks, 2, 79-102.

Holland, P. C. (1998). Temporal control in Pavlovian occasion setting. Behavioural Processes, 44, 225-236.

Kamin, L. J. (1965). Temporal and intensity characteristics of the conditioned stimulus. In W. F. Prokasy (Ed.), Classical conditioning: A symposium (pp. 118-147). New York: Appleton-Century-Crofts.

Kehoe, E. J., Horne, P. S., Macrae, M., \& Horne, A. J. (1993). Realtime processing of serial stimuli in classical conditioning of the rabbit's nictitating membrane response. Journal of Experimental Psychology: Animal Behavior Processes, 19, 265-283.

Kehoe, E. J., \& NAPIER, R. M. (1991). In the blink of an eye: Real-time stimulus factors in delay and trace conditioning of the rabbit's nictitating membrane response. Quarterly Journal of Experimental Psychology, 43B, 257-277. 
Lennartz, R. C., \& Weinberger, N. M. (1992). Analysis of response systems in Pavlovian conditioning reveals rapidly versus slowly acquired conditioned responses: Support for two factors, implications for behavior and neurobiology. Psychobiology, 20, 93-119.

Li, S. G., Lawler, J. E., Randall, D. C., \& Brown, D. R. (1997). Sympathetic nervous activity and arterial pressure responses during rest and acute behavioral stress in SHR versus WKY rats. Journal of the Autonomic Nervous System, 62, 147-154.

Mackintosh, N. J. (1974). The psychology of animal learning. London: Academic Press.

Marchand, A. R., \& Kamper, E. (2000). Time course of cardiac conditioned responses in restrained rats as a function of the trace CS-US interval. Journal of Experimental Psychology: Animal Behavior Processes, 26, 385-398.

McKenna, T. M., Weinberger, N. M., \& Diamond, D. M. (1989). Responses of single auditory cortical neurons to tone sequences. Brain Research, 481, 142-153.

McNish, K. A., Betts, S. L., Brandon, S. E., \& Wagner, A. R. (1997). Divergence of conditioned eyeblink and conditioned fear in backward Pavlovian training. Animal Learning \& Behavior, 25, 43-52.

Meredith, A. L., \& Schneiderman, N. (1967). Heart rate and nictitating membrane classical discrimination conditioning in rabbits under delay versus trace procedures. Psychonomic Science, 9, 139-140.

Pavlov, I. P. (1927). Conditioned reflexes (G. V. Anrep, Trans.). London: Oxford University Press.

Pearce, J. (1987). A model for stimulus generalization in Pavlovian conditioning. Psychological Review, 94, 61-73.

Prosser, S. (1995). Aspects of short-term auditory memory as revealed by a recognition task on multi-tone sequences. Scandinavian Audiology, 24, 247-253.
SAKURAI, Y. (1990). Cells in the rat auditory system have sensory-delay correlates during the performance of an auditory working memory task. Behavioral Neuroscience, 104, 856-868.

SмIтн, M. C. (1968). CS-US interval and US intensity in classical conditioning of the rabbit's nictitating membrane response. Journal of Comparative \& Physiological Psychology, 66, 679-687.

Supple, W. F., JR., \& Leaton, R. N. (1990). Lesions of the cerebellar vermis and cerebellar hemispheres: Effects on heart rate conditioning in rats. Behavioral Neuroscience, 104, 943-947.

VANDERCAR, D. H., \& SChNEIDERMAN, N. (1967). Interstimulus interval functions in different response systems during classical discrimination conditioning of rabbits. Psychonomic Science, 9, 9-10.

WAGNER, A. R. (1981). SOP: A model of automatic memory processing in animal behavior. In N. E. Spear \& R. R. Miller (Eds.), Information processing in animals: Memory mechanisms (pp. 5-47). Hillsdale, NJ: Erlbaum.

Wagner, A. R., \& Brandon, S. E. (1989). Evolution of a structured connectionist model of Pavlovian conditioning (AESOP). In S. B. Klein \& R. R. Mowrer (Eds.), Contemporary learning theories: Pavlovian conditioning and the status of traditionallearning theory (pp. 149-189). Hillsdale, NJ: Erlbaum.

Yin, H., Barnet, R. C., \& Miller, R. R. (1994). Second-order conditioning and Pavlovian conditioned inhibition: Operational similarities and differences. Journal of Experimental Psychology: Animal Behavior Processes, 20, 419-428.

(Manuscript received August 7, 2001; accepted for publication September 29, 2001.) 